\title{
A comprehensive study on HDR brachytherapy treatments of cervical cancers: using the first Co-60 BEBIG Multisource Unit in Bangladesh
}

\author{
Sadia R. Malik, MSBS, MS, PhD, Parvin A. Banu, MD, Naheed Rukhsana, MD \\ Radiation Oncology Division, Delta Medical College and Hospital, Dhaka, Bangladesh
}

\begin{abstract}
Purpose: The report presents an extraordinary synthesis of customer acceptance procedures (CAP), quality assurance tests (QA) in the treatment of cervical cancer patients, using the first Co-60 Multisource Unit ${ }^{\circledR}$ in Bangladesh. The QA and commissioning required measurements and emergency tests verifying the functional limits of parameters acceptable for the new HDR afterloader. Acceptable limits were: 1) the deviation between specified and measured source strength: $\pm 3 \%$; 2 ) the positional accuracy and uniformity: $\pm 1 \mathrm{~mm} ; 3$ ) the temporal accuracy (i.e. timer error and linearity and end error): $\pm 1 \%$ or $30 \mathrm{sec}$; 4 ) treatment planning system (digitizer and localization software): $\pm 3 \%$ or $1 \mathrm{~mm}$; 5) the distance from line to first dwell position and all the others: $5 \mathrm{~mm}$ and $10 \mathrm{~mm}( \pm 1 \mathrm{~mm})$.

Material and methods: Till February 2011, 47 patients were treated with HDR with more than 140 insertions applied. Amongst them, 12 patients were in stage IIB and IIIB, 22 were postoperative (IA and IB) while the remaining 13 patients were with unknown stage. All the cases with stage IIB and IIIB received concurrent chemo-radiation and brachytherapy. Postoperative patients received EBRT (50 Gy and HDR) according to the institutional protocol. CT scans were completed before HDR-plus planning with a good reproducibility $( \pm 2 \%)$ and were documented in repeating the plan for the same set up of a patient. Absorbed dose (Gy) to a point $P$, at a distance of " $r$ " in centimeters from a source of the Reference Air Kerma Rate (RAKR) has been utilized for the QA of the source, where source strength measurement was accomplished.

Results: All methods and analysis applicable to the QA and commissioning of Co-60 have been investigated and systematically analyzed, measured and documented before the treatment of a patient. Studies and safety requirements of this HDR remote afterloader were carried out. Acceptance and the QA were imperative to justify functionality and dependability in delivering the treatment. Implications of these studies were described in detail in this paper, where equipments and guidelines of measurement parameters are enunciated.

Conclusions: We noted that contouring structures from CT images, prescription points for dose delivery, optimization, isodose evaluation, DVH, dwell times and a 3-D Dose reconstructed images, etc. followed by a final verification after delivering the treatment at the console, are well prepared in the new planning software. We present our material as an early preliminary report.
\end{abstract}

Key words: HDR brachytherapy, cervical cancer, Co-60, MultiSource ${ }^{\circledR}$.

\section{Purpose}

Brachytherapy, as a procedure, started since Roentgen discovered X-rays in 1895 followed by Becquerel's discovery of natural radioactivity in 1896, when he reported the destructive impact of radioactive isotopes. The first nasopharyngeal cancer was treated by Dr. Voigt of Hamburg and the first afterloading was applied by an American surgeon, Dr. Robert Abbe in 1905. In brachytherapy (also referred to as curie-therapy) radioactive substance is encapsulated and placed directly or near the tumour. The sources are located into the treatment volume. High-dose-rate (HDR) brachytherapy is a technique using a source of specific high activity (10 Curie Ir-192 source or 2 Ci Co-60 source) in delivering a therapeutic dose of radiation, using temporarily placed needles, catheters or other applicators to the respective procedures. HDR brachytherapy was proven to be highly successful treatment for cancers of prostate, cervix, endometrial, breast, skin, bronchus, oesophageal, and head and neck. Soft tissue sarcomas, ocular melanomas and several other types of cancer could also be treated with success. Any tumour that is accessible to needles, catheters or tubes is potentially treatable [1-5]. Implant techniques that was applied at Delta Medical College and Hospital are described in our paper, following the QA, commissioning and 
subsequent applications in developing treatment plans and treatment delivery.

About $18 \%$ of female patients are referred to our hospital with the diagnosis of cervical cancer. Low socio-economic condition and infection with Human Papilloma Virus are the causative factors of high incidence of cervical cancer in this region. HDR treatment facility is now available to treat such patients. The fundamental need to treat such patients has made it mandatory for an accurate and reproducible system for dose delivery which was achieved and described explicitly in this paper in order to provide a guideline to other users in any other clinics and hospitals.

\section{Material and methods}

The source is usually attached to (or embedded in) the end of a wire, that drives the source into the applicators that have previously been placed. The source dwells in the preplanned positions, for a present time, before stepping along the catheter. This process is repeated in order to create the required dose distribution in the treatment plan. By varying the position and dwell time, the dose is optimised to adapt a dose geometry that is compliant to the shape of the target. In general, the patient receives the total prescribed dose in a series of 2-5 fractions. The steps to set up the patient and deliver the treatment are briefly described below after the acceptance of HDR unit. The initial QA needs to be evaluated. The breakdown of the procedures are stated in the following steps [6].

\section{Quality assurance and acceptance [7]}

\section{Measurement of source strength}

The source strength measurements were performed using a well-type ionization chamber (PTW type 33004-00297) or a thimble ionisation chamber (T48012) using PTW Unidos Electrometer. The constancy of the well chamber and thimble chamber was checked before and after the source strength measurement using Cs-137 and Sr-90 check sources, respectively. In order to compare the source certificate data, all measurements were decay - corrected to the manufacturer's source calibration data at a reference laboratory. The well chamber was supplied with the PTW calibration factors of the Ir-192 sources used in the Nucletron Microselectron ${ }^{\circledR}$, MDS Nordion Gammamed ${ }^{\circledR}$, Vari-

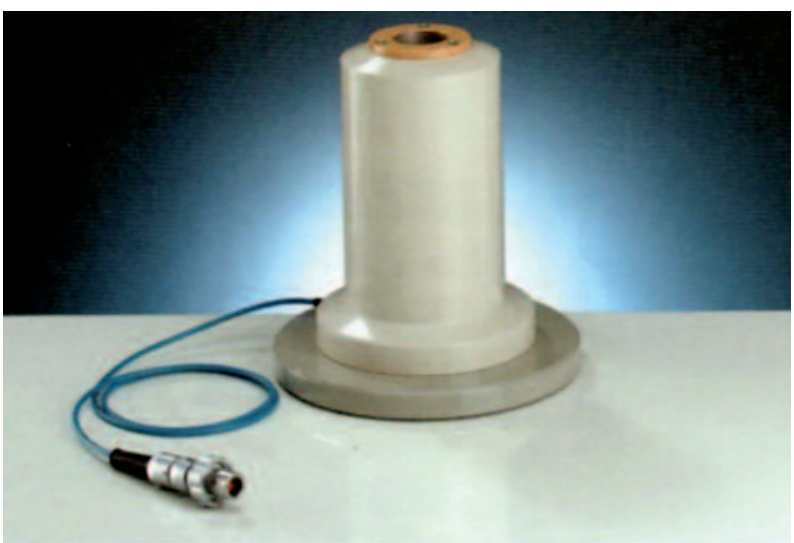

Fig. 1. Ionisation chamber for dosimetry an Varisource ${ }^{\circledR}$ and BEBIG Multisource ${ }^{\circledR}$ HDR systems. For each of these, the calibration factors were provided for the calculation of the Reference Air Kerma Rate (RAKR) (in $\mathrm{m} \times \mathrm{Gy} \times \mathrm{h}^{-1}$ at $1 \mathrm{~m}$ ) or apparent activity (Ci or GBq). The well chamber measurements were made with 1400 universal applicator transfer tubes (IBt Bebig), using the calibrated chamber insert and locking ring. The point of maximum response for the chamber was first determined by stepping the source from the distal end in $5 \mathrm{~mm}$ step sizes for a distance of $12 \mathrm{~cm}$, using a dwell time of $10 \mathrm{~s}$ per position. Source strength measurements were then performed at the point of maximum response and the relevant calibration factors were as follows:

$$
\mathrm{S}=\mathrm{R} \times \mathrm{CF}_{\text {cal }} \times \mathrm{CF}_{\text {ion }} \times \mathrm{CF}_{\mathrm{T}: \mathrm{P}}
$$

Where, $S$ is the source strength $\left(\mathrm{m} \times \mathrm{Gy} \times \mathrm{h}^{-1}\right.$ at $1 \mathrm{~m}$ or $\mathrm{Ci}$ or $\mathrm{GBq}), \mathrm{R}$ is the leakage corrected electrometer reading $(\mathrm{mA}), C F_{c a l}$ is the chamber calibration factor, $C F_{\text {ion }}$ is the correction for ion recombination and $C F_{T: P}$ is the air mass correction.

\section{Measuring in a well type chamber}

We used a well type Ionisation Chamber for Dosimetry (Fig. 1). Advantages: 1) easy to handle, 2) good geometric reproducibility, 3) less sensitive to backscattered radiation from the surrounding. Disadvantages: 1) only usable for all source calibration, 2) extra cost for expensive chamber. In Table 1 we present characteristic of the source used in the HDR Unit [8].

Determination of isotope purity of the source (QA)

Method: Repeating the measurements in phantom well chamber and comparing the results with the half-life corrected value at the beginning. Time scheme: 1 ) daily before $T_{x}, 2$ ) in the first 6 months: every month, 3) afterwards: every half a year.

\section{Positioning of the source}

Figures $2 \mathrm{~A}$ and $2 \mathrm{~B}$ presents the methods for positioning of the source as a routine QA before treatment of the day.

\section{Measuring the dose distribution}

Brachytherapy characteristic (step fall-off the dose) are shown in Fig. 3.

\section{Acceptance test}

The experimental determination of RAKR was evaluated by means of a calibrated well type chamber and associated equipments. The equipment: Ionization chamber T33004 calibrated for Ir-192 and/or Co-60. The accessories used: source holder for $3 \mathrm{~mm}$ diameter applicator type 33004.1.013, the electrometer: Unidos ${ }^{\circledR}$ or Multidos ${ }^{\circledR}$.

\section{QA test: treatment planning system}

We used digitizer and localization software (tolerance: $3 \%$ or $1 \mathrm{~mm}$ ), calculation algorithm (tolerance: $3 \%$ or $1 \mathrm{~mm}$ ).

\section{QA test: radiation safety}

Requirements were the following: 1) Gamma Zone Monitor, 2) CCTV, 3) unit leakage. We accepted the follow- 
Table 1. Source specifications for Co-60 used at HDR unit [9]

\begin{tabular}{ll}
\hline Cobalt-60 & \\
\hline For use with afterloader & Multi Source/Gyne Source (SN > 100) \\
\hline Application & Gamma-source for HDR brachytherapy \\
\hline Type & Afterloading - radiation source \\
\hline Mark & Co $0 . A 86$ \\
\hline ISO classification & ISO $2919-1998 / C$ 65444 \\
\hline Construction & Single encapsulation Cobalt-60 pellet tightly welded in a stainless steel capsule \\
\hline Material of capsule & 1.4576 \\
\hline Radionuclide & Co-60 \\
\hline Half-life & 5.27 years \\
\hline Physical-chemical form & Solid, metal \\
\hline Content activity & 70 GBq $\pm 10 \%$ \\
\hline Recommended working life & 100 000 source transfer or max. 5 years with afterloader under normal \\
& conditions and used with original Eckert \& Ziegier BEBIG applicators \\
\hline External measurements of source & Diameter: $1 \mathrm{~mm}$ \\
& Length: $2180 \mathrm{~mm}$ \\
\hline Measurements of active part & Diameter: $0.5 \mathrm{~mm}$ \\
& Length: $3.5 \mathrm{~mm}$
\end{tabular}

\section{A}

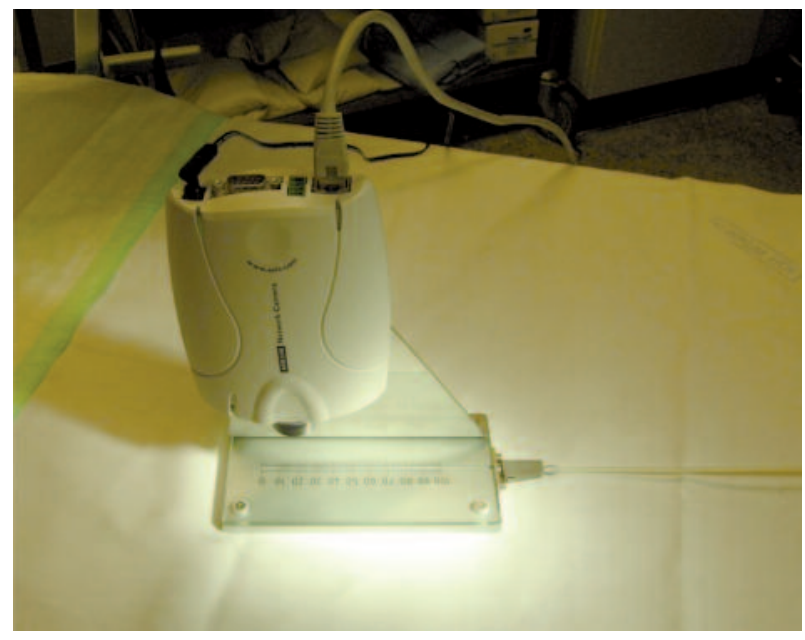

B

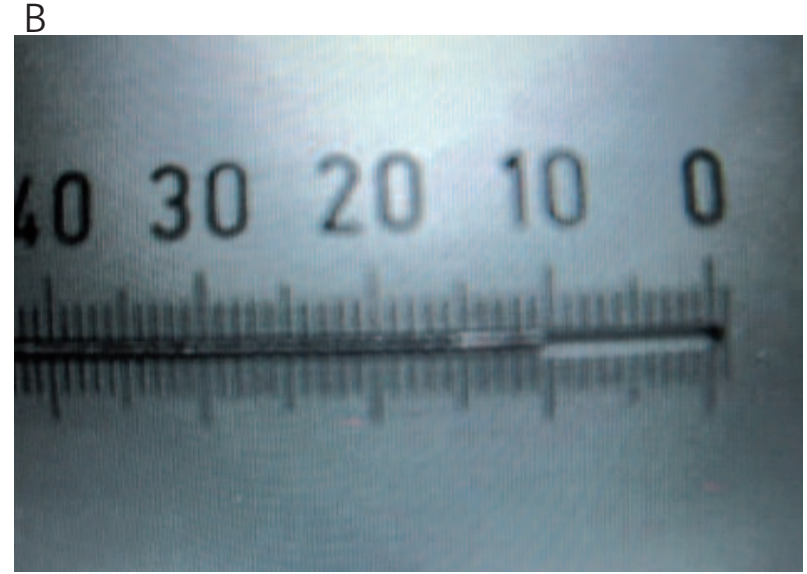

Fig. 2. Visual test of source positioning with the use of a camera

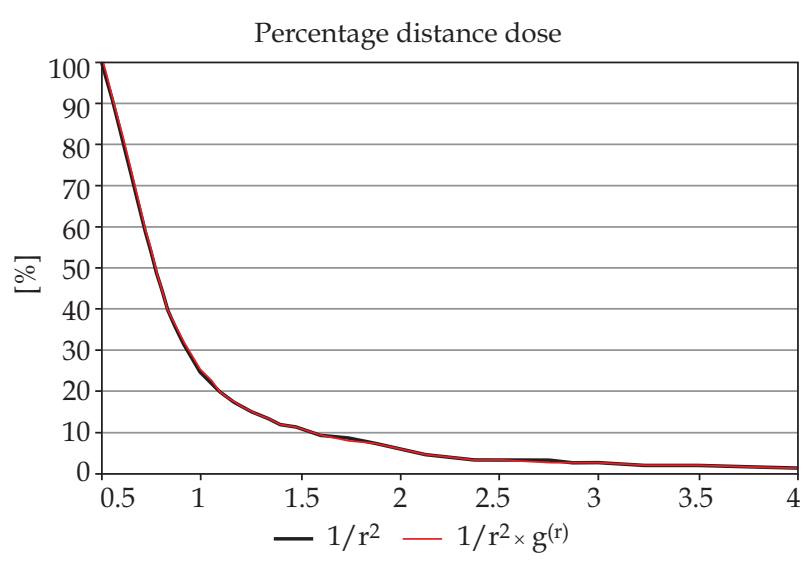

Distance from the source $(\mathrm{cm})$

Fig. 3. Dose fall off with distance

ing standards: 1$)$ measured dose rate $\left(\mu \times \mathrm{Gy} \times \mathrm{h}^{-1}\right)$ at $5 \mathrm{~cm}$ from the surface of the source storage (tolerance: $\leq 100 \mu$ $\left.\left.\times \mathrm{Gy} \times \mathrm{h}^{-1}\right), 2\right)$ measured dose rate $\left(\mu \times \mathrm{Gy} \times \mathrm{h}^{-1}\right)$ at $1 \mathrm{~m}$ from the centre of the source storage (tolerance: $\leq 10 \mu \times \mathrm{Gy}$ $\times \mathrm{h}^{-1}$ ). Information on emergency procedures must include the contact numbers of the responsible personnel and displayed at treatment console of the HDR unit.

\section{Treatments}

\section{Patient set up procedure}

Patients with the diagnosis of cervical cancer attended oncology outpatient department at Delta Hospital Ltd. The examination for staging was done under local anaesthesia, after which the treatment decision was made according to the institutional protocol. As a preparation for brachytherapy, enema was applied and the procedure was 
mostly completed under general anaesthesia. After cleaning and dressing, the catheterization was done with $10 \mathrm{cc}$ of air introduced in the balloon in order to localize the bladder. After the applicators were set up, the CT simulation was performed by Somatom Emotion-16 Slice Scanner ${ }^{\circledR}$.

\section{Principles and treatment planning of HDR}

\section{brachytherapy [9]}

Basis of BT physics, the advantages may be summarised as: 1) short distance treatment, 2) rapid fall off-dose, 3) sparing of critical organs, 4) dosimetry at different geometry, detector, equipment, calibration, etc., 5) dose prescription depended on technique.

Key aspects of BT planning included: optimal target dosimetry, sparing organs at risk (OaRs), preventing high toxicity levels, shortest time-planning delivery, accurate dose conformity and homogeneity. The HDR planning is quite different from conventional external beam radiation therapy planning. One single high activity miniature source mimics the line sources; isodose distribution depends on dwell positions and dwell times. The dwell positions times are determined by the constraints specified during planning: tumour dose and critical organ dose such as maximum dose and volume of the OaRs.

\section{Dosimetry [1]}

Dose rate at a point depends on: RAKR, the source shape, composition and thickness of its metallic sheath, composition of means between the source and a point [10].

\section{Advantages of use of RAKR}

RAKR specifies the strength of a source. The source strength is directly traceable to a national standard, the dose rate in a tissue is closely related to the RAKR for a point source and it is easy to estimate hazards around an application.

\section{Imaging in 3D}

Implant target and other anatomical structures are not usually identifiable on radiograph. To overcome this, 3D sectional imaging is becoming increasingly common where CT and MRI modalities are used. The advantages of the use of sectional CT imaging are: 1) no projection images are needed and the problem of matching sources from projection image to projection image is avoided, 2) cross sectional isodose can be directly superimposed onto the corresponding sectional CT image i.e. related to the target volume and surrounding anatomy, 3) structure specific dose where volume histograms can be calculated. A major disadvantage is that the CT scan must be done with the applicator in situ.

\section{Computer dose calculations}

Computations of semi 3D (multiple planes) and 3D (Volume) calculations consist of summing up the dose contributed by each source at grid points arranged in 3D images. The dose distributions that are calculated in planes parallel to the projection images, can be scaled and superimposed onto the projection images (radiographs). Caution should be exercised as the dose distribution in any one plane represents only a very narrow segment (section) of the implant and the distribution can be quite dissimilar in a slightly different plane. Dose volume histograms (DVH) refer to summation of the dose received by each of the structures of interest. DVH shows how much of the volume of each structure receives prescribed dose.

\section{Types of DVH}

1) Cumulative DVH - is most useful when comparing the overall behaviour of a number of different structures for one particular treatment plan. 2) Differential DVH - more relevant to understand in detail the shape of the histogram. 3) Natural DVH - a particular variation of the source specific differential DVH - influence of the inverse square law is suppressed.

\section{Dose volume histogram optimization}

The DVH-shaper is a unique graphical user interface which enables to interact with the optimization engine, either VBO or DVHO, directly through the DVH graphs. It offers a user friendly interface, allowing interactive optimization while considering dose or weight penalties, or other important factors. The user simply sets the adaptation of a DVH curve for any target or the OaRs. The system then takes care of the calculations itself. The DVHshaper, in a combination with the "history" and "lock" functions, offering a unique and secure platform for interactive DBH-based optimization, minimizing the learning curve of the user.

Disadvantages: 1) There is little clinical data available on the relation between dose-volume and resulting effect i.e. on the probability of well-defined endpoint occurrence. 2) Another aspect of the effect of dose - volume relations particularly in brachytherapy, is the contiguity of high dose volumes in normal tissues, if these normal tissues lay inside the PTV (especially in prostate HDR brachytherapy).

\section{Isodose calculation}

Different kind of dose control helps to optimize the shape of the distribution: setting the dwell-times graphically or by number; setting of the dose $\left(R_{x}\right)$ at one control point (Manchester ' $A$ ' point), the pattern of the dwell positions remains unaffected. We can use: 1) control point optimization, 2) geometrical optimization, 3 ) dose point optimization where the inverse planning is setting up the dose limiting points and optimizing with a predetermined criteria, 4) isodose shaping allowing interactive isodose modelling by a mouse operation for the coverage of the target.

The optimization was completed by manually defined control positions (Fig. 4). Our reporting tools were as follows: patient information, source data, dwell times and positions, cumulative and differential dose volume, isodose views and $R_{x}$ in dose, length, diameter, etc. Checking was done by qualified personnel. Examples of isodose distribution and DVH graphs are presented in Figs. 5A and 5B.

\section{Results}

Since December 2010 to February 2011, the cylinder, tandem and ovoid or the cylinder and tandem treatments were 

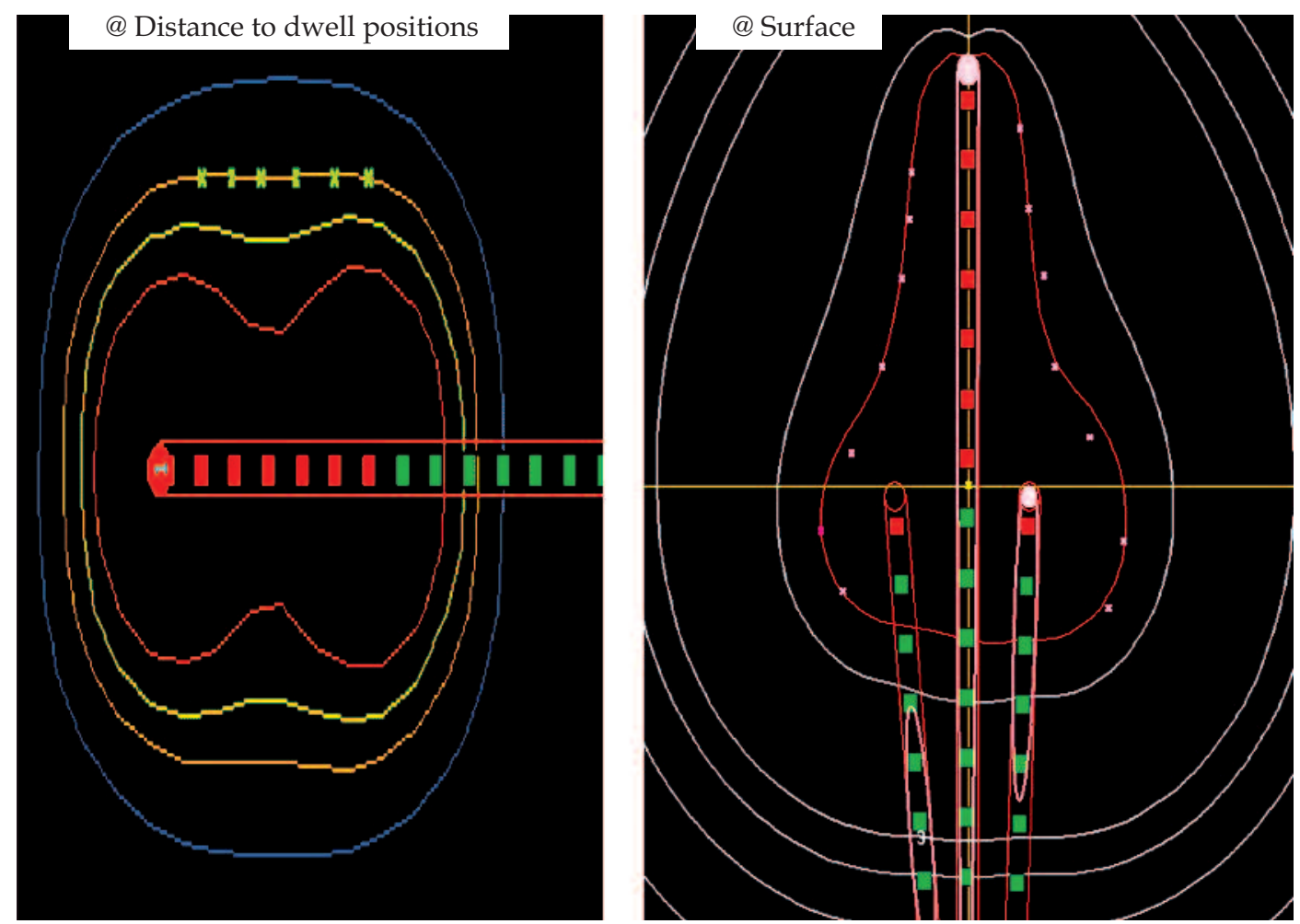

Fig. 4. Dose optimization using the Cylinder \& Fletcher Unit
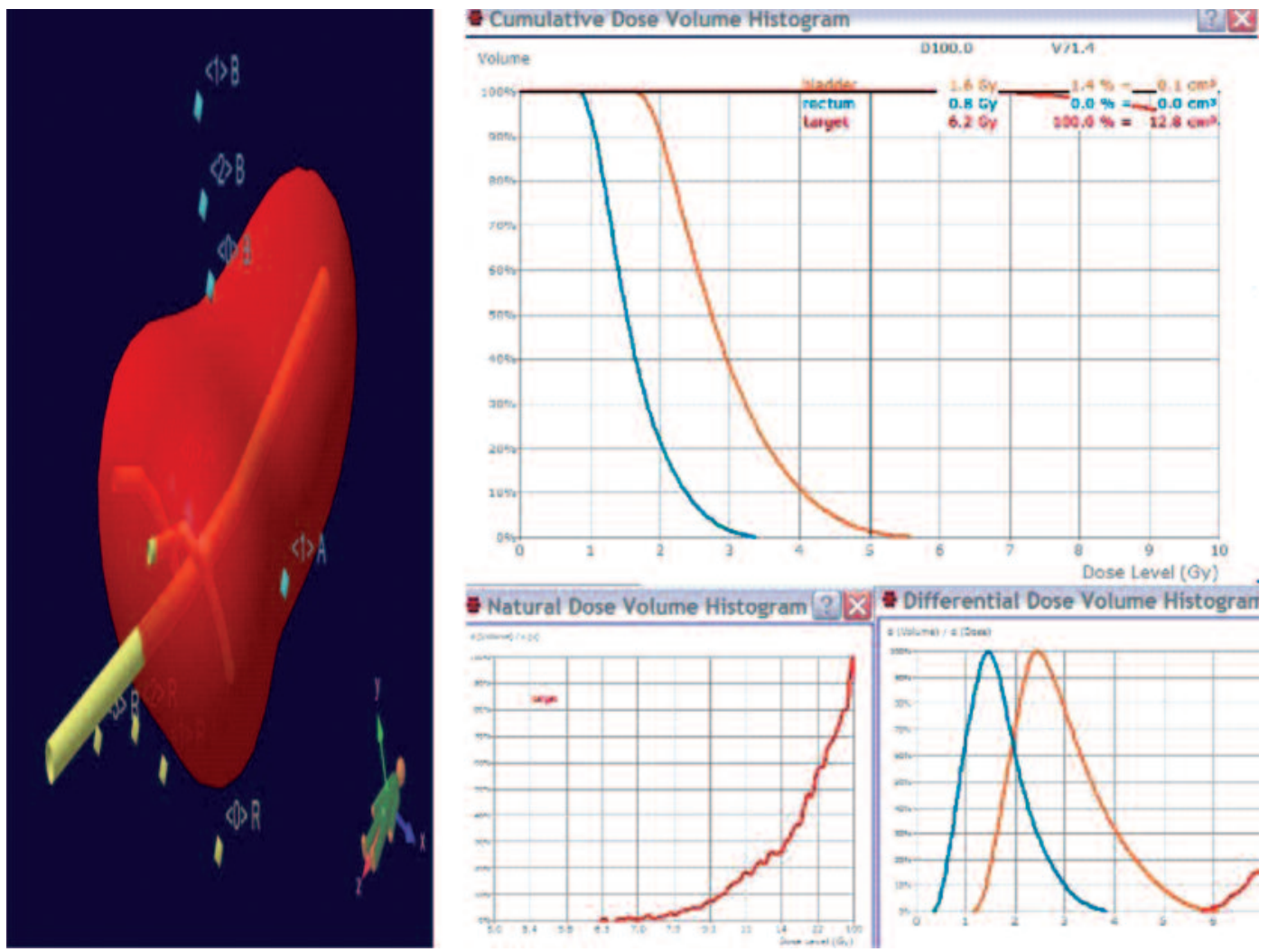

Fig. 5A. Isodose distribution \& DVH graphs 


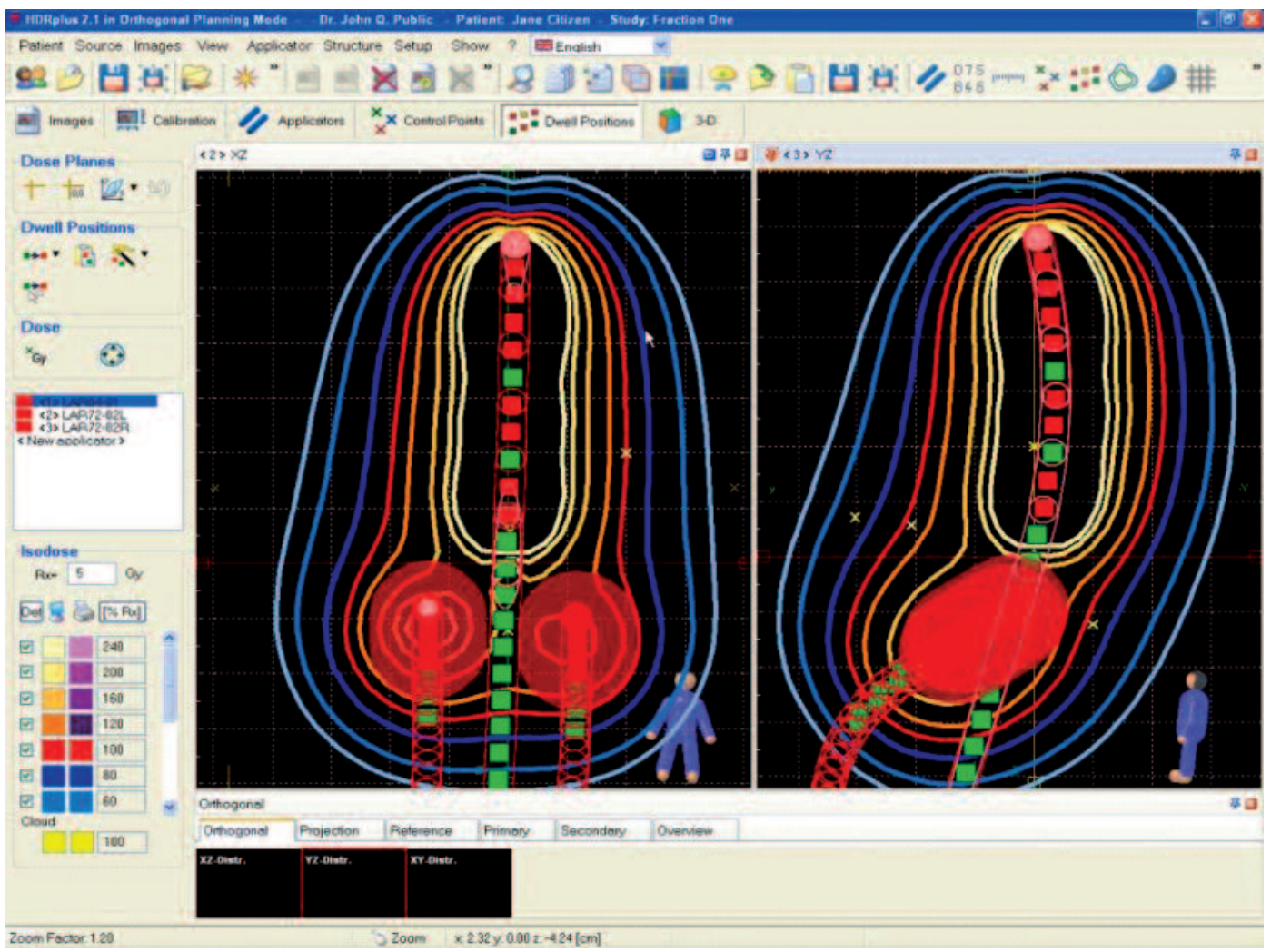

Fig. 5B. Isodose distribution \& DVH graphs

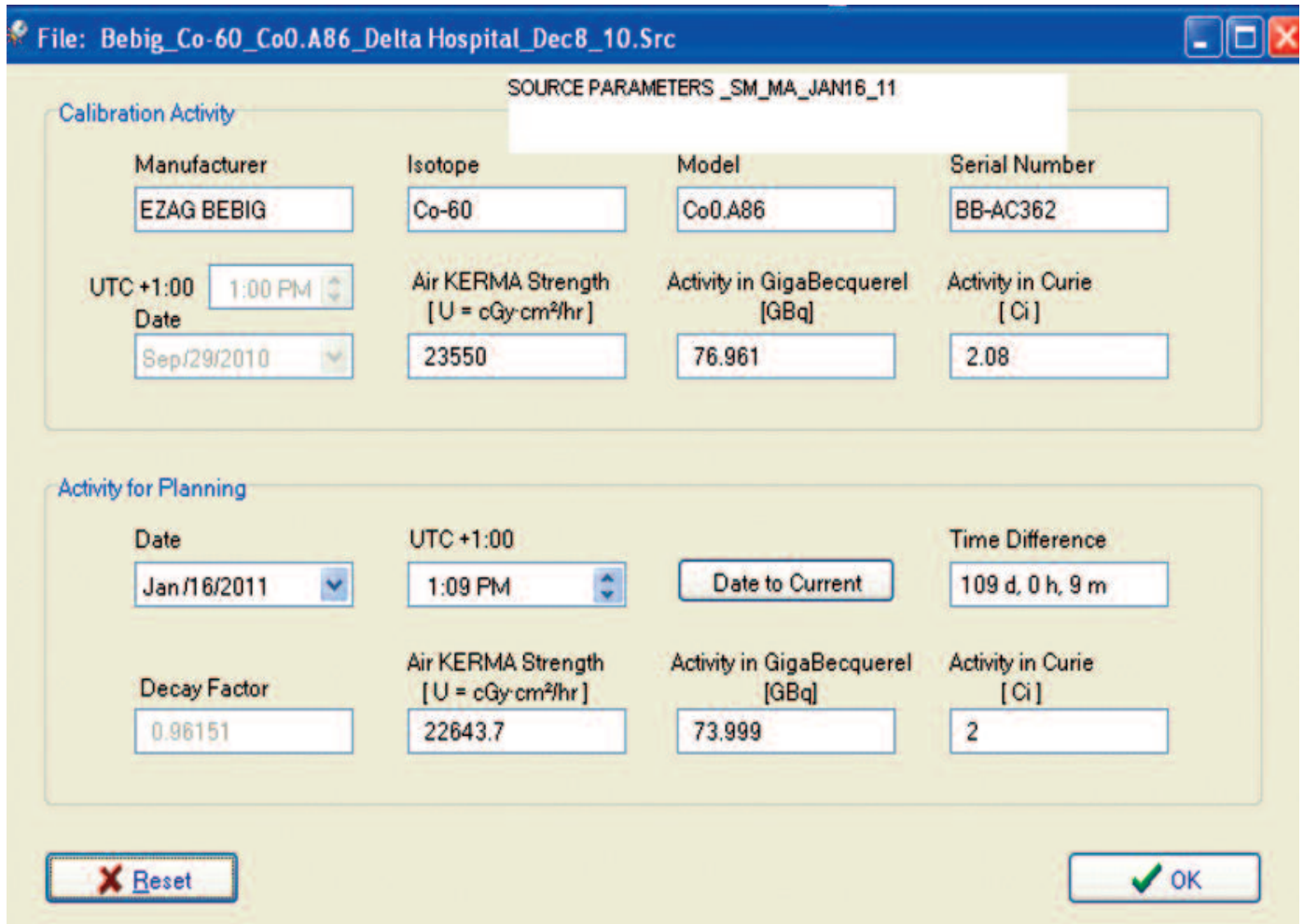

Fig. 6. QA source data before treatment plan evaluation 

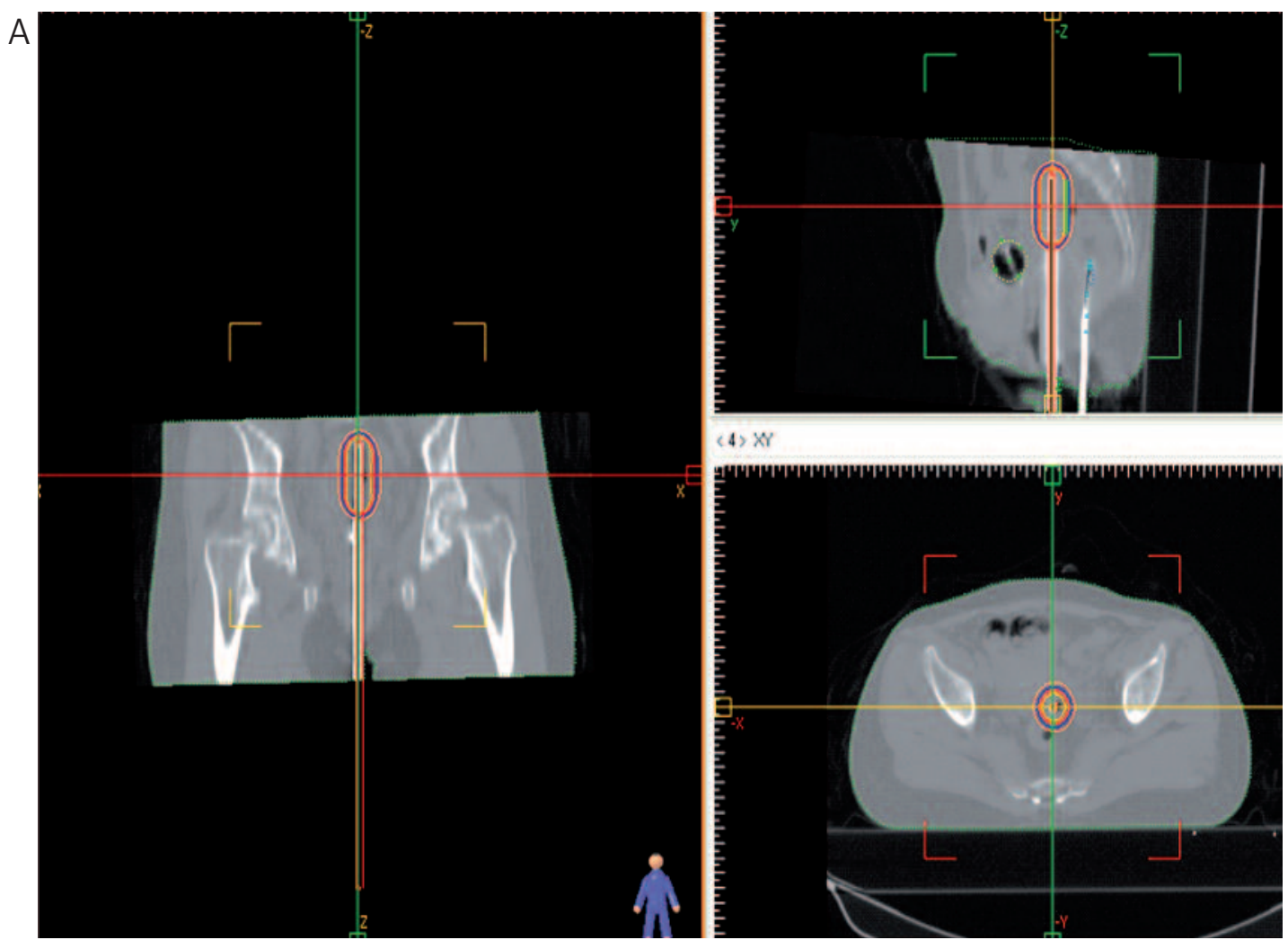

(4) $X Y$
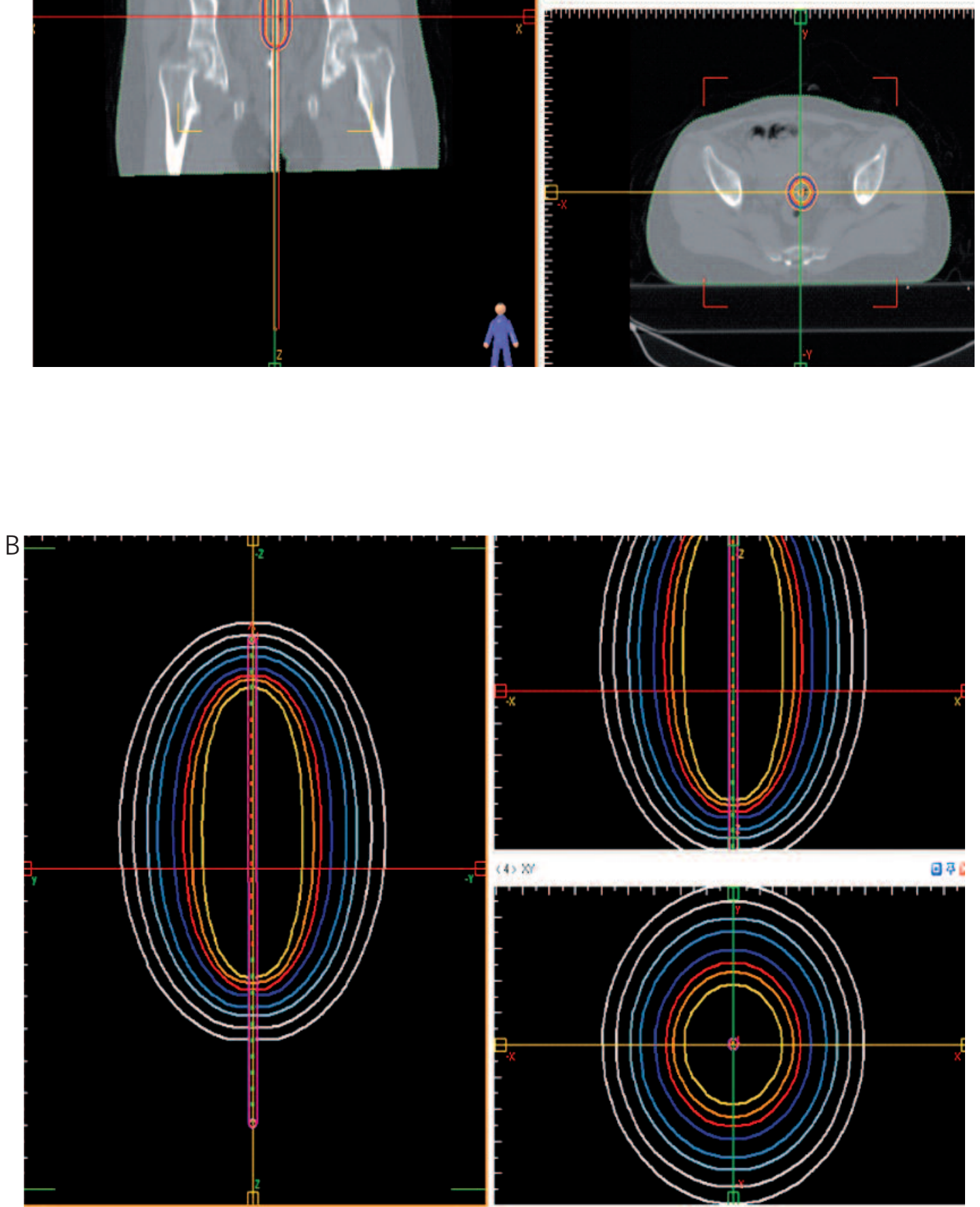

(4) $x$

অ\$द

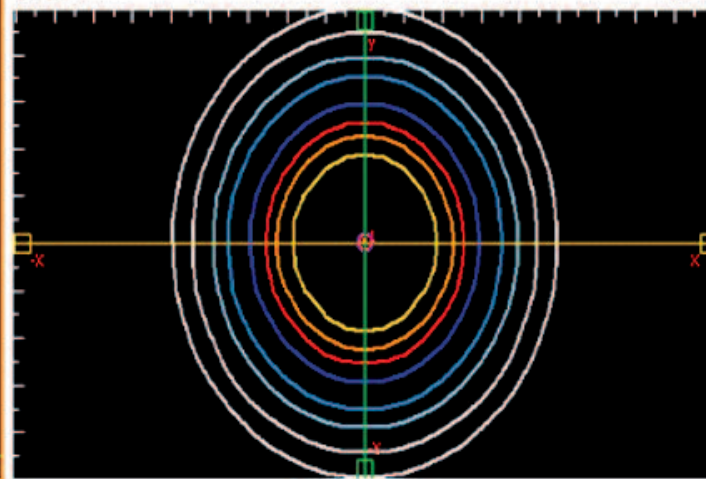

Fig. 7A-B. Isodose distribution from a CT based cylinder plan 
C

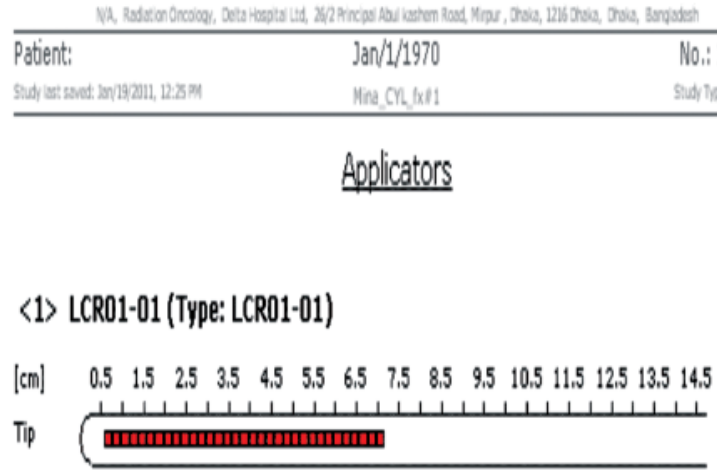

Fig. 7C. Dwell points showing Rx length in an applicator for a cylinder

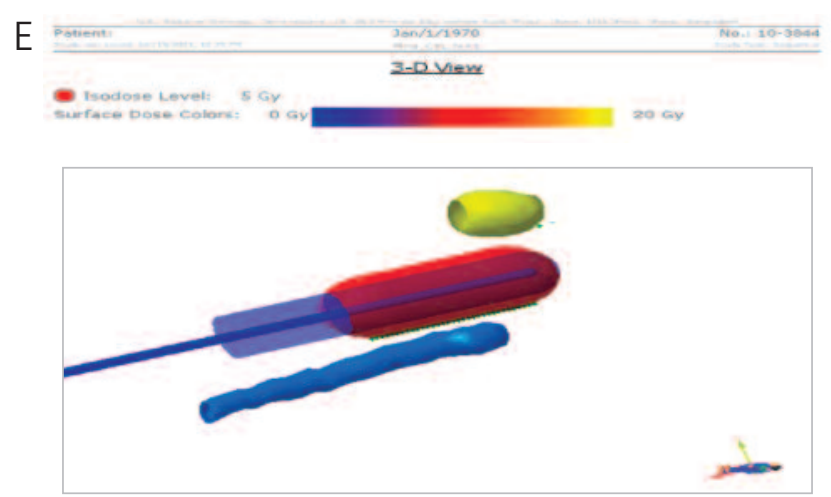

Fig. 7E. 3D view of isodose distribution in a cylinder plan

D Relative volume

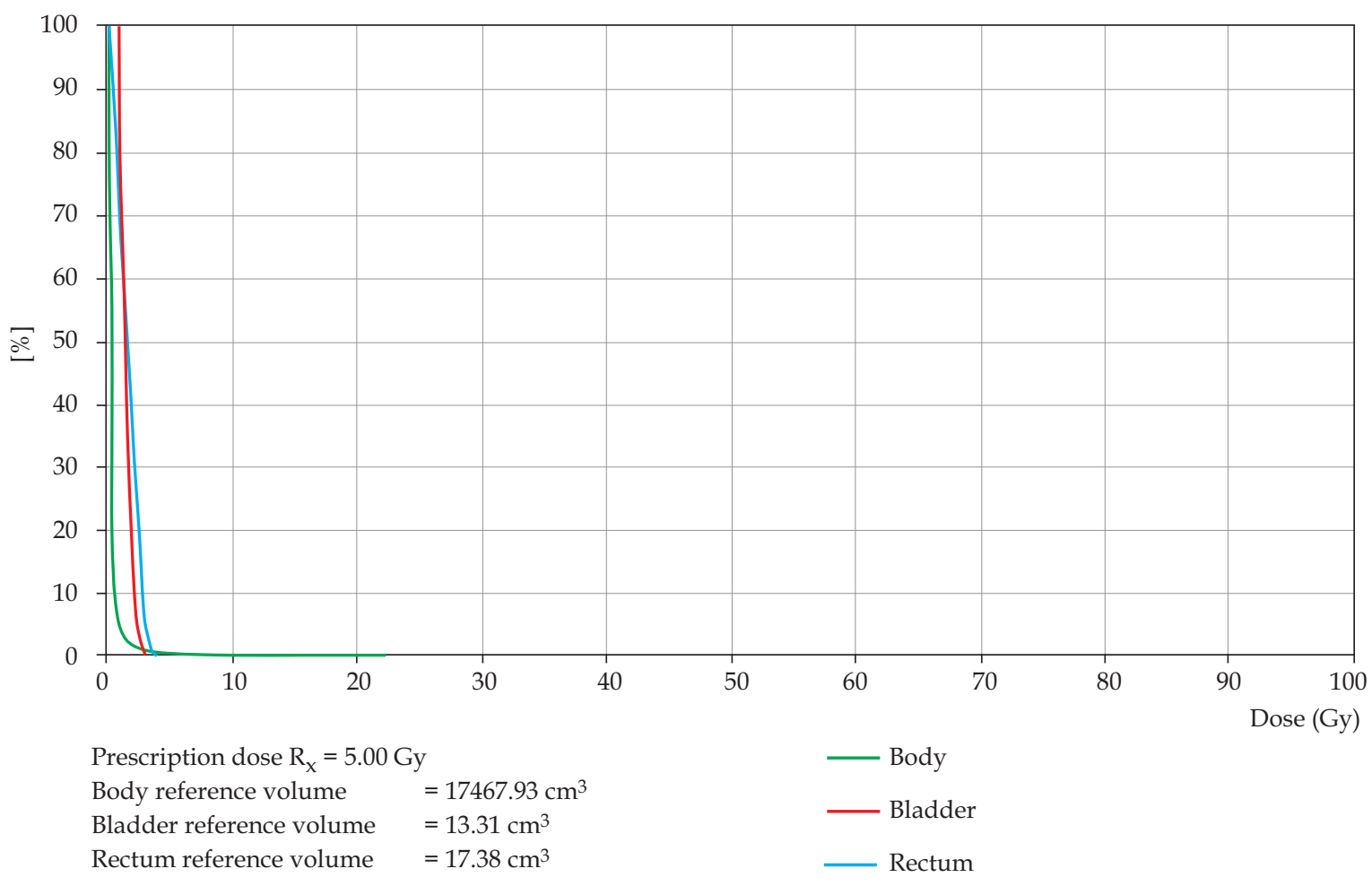

Fig. 7D. Dose Volume Histogram (DVH) for a cylinder applicator

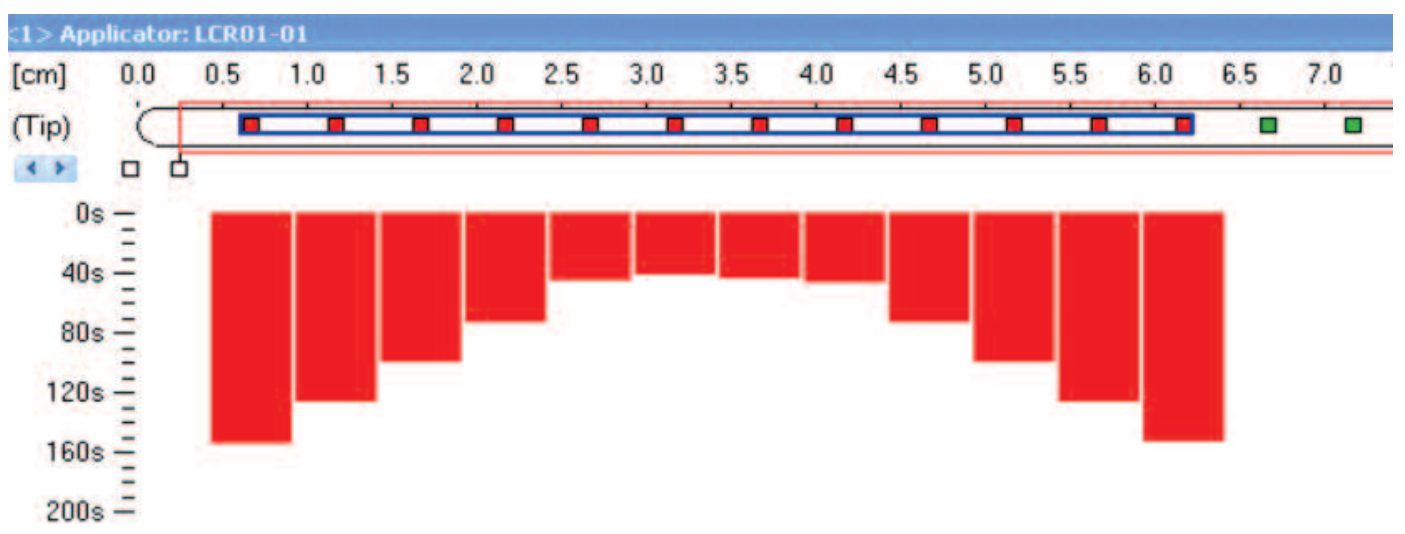

Fig. 8. View of optimised dwell point positions of the source 

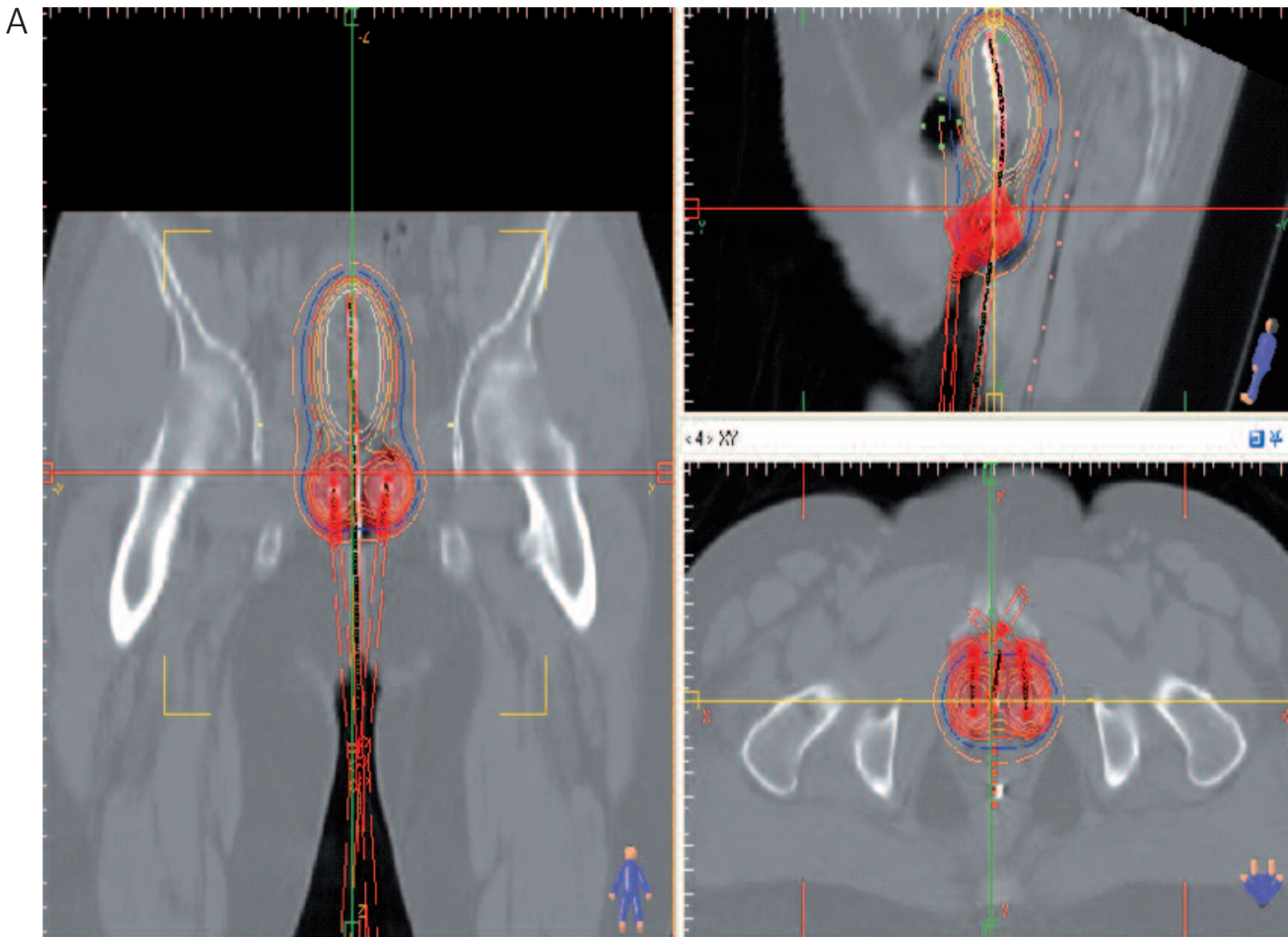

Fig. 9A. CT - image based planning for the set up of tandem and 2 ovoids showing points A and B with bladder and rectum sites

\section{B}

\begin{tabular}{ccc}
\hline Patient: Trial, ARCHONA_fx $\$ 22$ & Jan $/ 2 / 1955$ & No.: 00000 \\
TAO tet & Applicators
\end{tabular}

\section{<1> LCT82-02L (Type: LCT82-02L)}

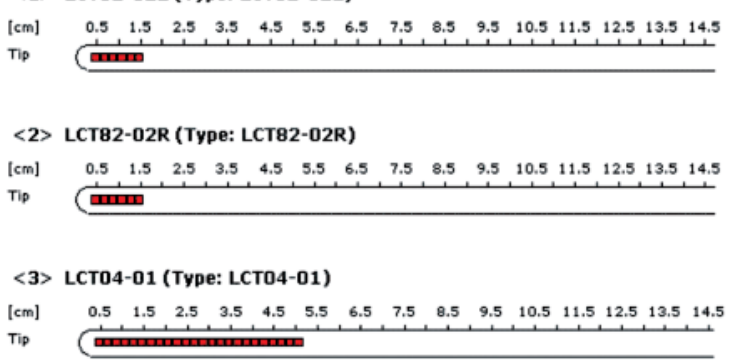

C
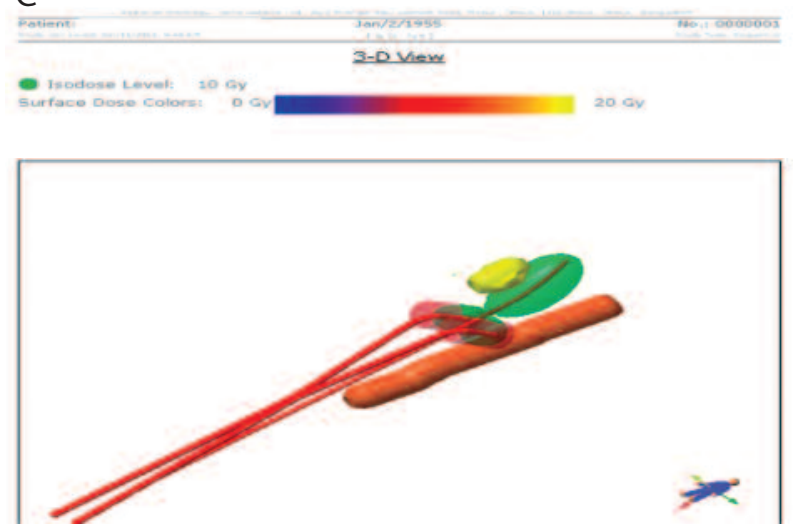

Fig. 9B. Dwell points for a source positions in 2 ovoids and a tandem. C) 3D view of dose distributions in a tandem and 2 ovoids along with bladder and rectum

carried out using CT scans by Somatom Emotion spiral CT scanner ${ }^{\circledR}$ followed by planning with the use of CT image sequences. In Fig. 6, the source QA data are presented. Isodose distribution from the cylinder based plan are shown in Figs. 7A-E. Patients reports was verified and signed by the radiation oncologist and a physicist. Every report included all other information such as applicator length, $\mathrm{R}_{\mathrm{x}^{\prime}}$ $\mathrm{DVH}$, dwell positions, bladder and rectum doses and source data. Figure 7C depicts the type of cylinder applicator and the prescribed length for treatment. Figures 7D and 7E presents the DVH and a view of isodose distribution in a cylinder. In Fig. 8, a view of dwell point source position which was optimised for the plan is shown. Figure 9A presents the anatomy of CT-views with the isodoses for a treatment plan using a tandem and two ovoids. Figures 9B and 9C shows dwell point source positions and control points $\mathrm{A}$ and $\mathrm{B}$ along with the rectum and bladder doses for a $3 \mathrm{D}$ view of dose distributions for tandem and ovoid plan, respectively. Figures 10A and 10B presents characteristic of patients treated at Delta Hospital Ltd. using the first BEBIG Co-60 Multisource ${ }^{\circledR}$.

\section{Discussion and conclusions}

This paper outlines the procedures and the steps to follow in the implementation of the Multisource Unit $₫$ in cli- 

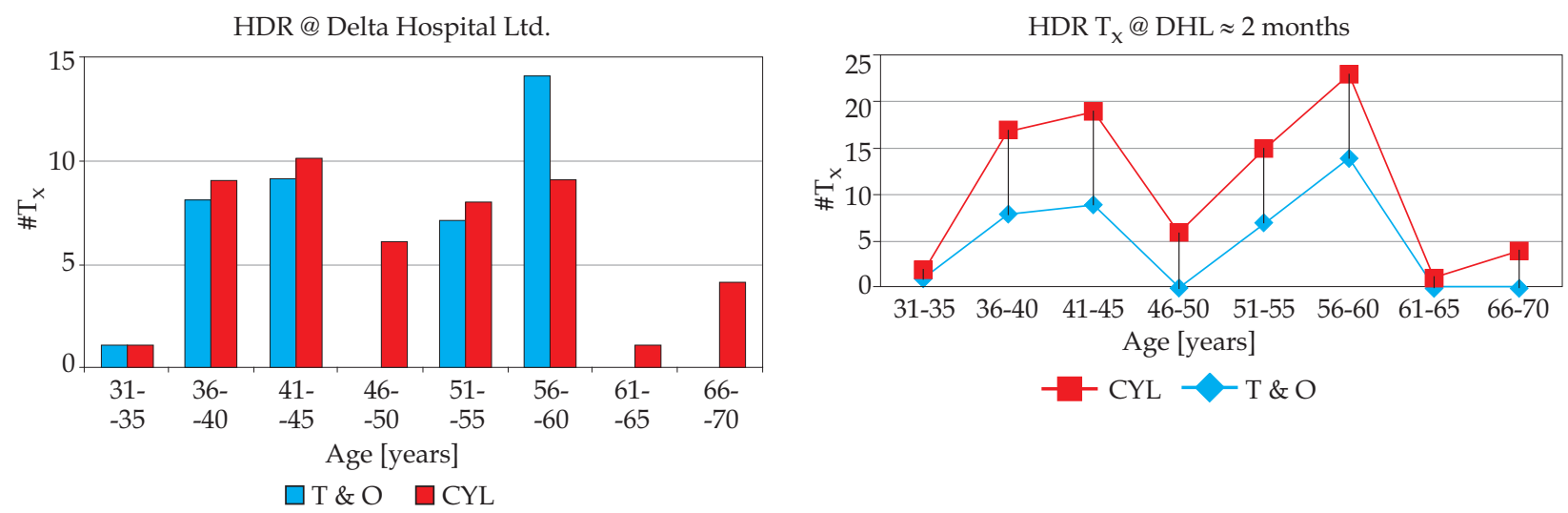

Fig. 10. Variation of number of cervical cancer patients at Delta Hospital Ltd. (December 10 to February 28), using BEBIG ${ }^{60}$ Co Multisource with the Fletcher \& Cylinder (total $t_{x}=87$ )

nical use. This work emphasised the fact that if acceptance and the QA tests are rigorously completed, then any user will acquire no difficulties in delivering the HDR treatments. The limits of acceptability, as documented earlier, should be achieved by the users in other institutions. This paper, therefore, provides a basis for the first users from evaluation of parameters to clinical applications. Low dose rate (LDR) brachytherapy with Cs-137 is inconvenient for the patients, considering long treatment time and additional rectal and bladder complications, as seen in long follow up of our experience. The treatments delivered by this HDR unit reported no adverse complications, so far, as the doses to critical structures such as bladder and rectum were low in our treatment plans. Study of the cervical cancers patients demonstrates that the system is reliable, if the QA, acceptance and dosimetry are carefully evaluated in repeating treatment plans and in using various applicators with multiple trials in patient's CT images. The measurements on source data are within the tolerance limits and are traceable to a reference laboratory in Germany. The potential of using the unit and the software will be explored in other anatomical sites. We have found the system performance acceptable in cervical cancer. The bladder and rectum doses are well below the acceptable limits as recommended by the ICRU.

\section{Acknowledgment}

Our sincere thanks to Delta Hospital Ltd. and Professor S. Mukarram Ali, FRC Path, for his encouragement in providing the facilities to work on HDR treatments as a first user in Bangladesh. We appreciate the clinical support of J. Yasmin, MBBS, M. Phil, and Mushfiqa Ahmed, MS.

\section{References}

1. Aird EGA, Jones $\mathrm{CH}$, Joslin $\mathrm{CAF}$ et al. Recommendations for brachytherapy dosimetry. British Institute of Radiology, London 1993; 1-19.

2. Nath R, Anderson LL, Luxton G et al. Dosimetry of interstitial brachytherapy sources. Med Phys 1995; 22: 209-234.

3. Rivard MJ, Coursey BM, DeWerd L et al. Update of AAPM TG-43 report: a revised AAPM protocol for brachytherapy dose calculations. Med Phys 2004; 31: 633-674.

4. Kubo HD, Glsgow GP, Pethel TD et al. High dose-rate brachytherapy treatment delivery. Med Phys 1998; 25: 375-403.
5. Ravinder N, Howard A, Charles C et al. Intravascular brachytherapy physics. Med Phys 1999; 26: 119-152.

6. PTB (Physikalisch-Technische Bundesanstalt) Calibration certificate for NE261 ionization chamber in connection with a PMMA phantom (Krieger-Phantom) 2009; Ref. No. 6.2212/09K, unpublished.

7. Andrassy M. Acceptance Test of HDR - Afterloading Sources 2009 TD09_085, Eckert \& Ziegler BEBIG GmbH, unpublished.

8. Ibt BEBIG Handbook 2010; Revised June 10. Berlin, Germany.

9. Hoskin P, Coyle C. Radiotherapy in practice: brachytherapy. Oxford University Press, Oxford 2007; Chapter 2: 21-41.

10. The GEC ESTRO Handbook of Brachytherapy. Gerbaulet A, Potter R, Mazeron J-J et al. [eds.]. ESTRO, Bruksela 2002. 